\title{
Measurements of Erythrocyte Flow Velocity by Means of Grating Laser Microscope
}

\author{
Junko NitTa,* Tomiyasu Koyama, Yuji KıKuchI, \\ and Yoshio SHINDo \\ Research Institute of Applied Electricity, Hokkaido University, \\ Sapporo, Hokkaido, 060 Japan
}

\begin{abstract}
The possibility of measuring erythrocyte flow velocity by means of a grating laser microscope has been examined in an in vitro test using erythrocytes smeared on glass plates and in vivo using microvessels in the web of a frog foot. Magnified projections of flowing erythrocytes are spatially filtered by a simple glass plate grating and then detected by a photomultiplier. Output signals from the photomultiplier are first electronically filtered so as to yield oscillating burst-like wave signals. Moving erythrocytes resulted in burst-like oscillation in output signals whose wave period $(T)$ changed with their velocities. These results suggest that the velocity $(V)$ of erythrocytes could be given by $V=d / M T$, where $d$ and $M$ represent the grating constant and the optical magnification, respectively. The requirement for the establishment of this relation was examined by the microscopic observation of smeared erythrocyte preparation. It was shown that the flow velocity of erythrocyte could be measured even when the size of magnified images of erythrocytes strongly exceeded the value of $d$ and even when many erythrocytes existed. A preliminary experiment to confirm the possibility of methodological adaptation was made by measuring the flow velocity in venules of foot web of frogs. Heart rate increased in all six frogs studied when the frog body was warmed. However, the peripheral flow velocity increased only in three frogs, while it remained almost unaffected in the other three. This result suggests that the frog peripheral microcirculation is affected by complicated intrinsic factors.
\end{abstract}

Key Words: grating laser microscope, erythrocyte flow velocity, microvessels, temperature, frog foot web.

The crossed-dual laser Doppler microscope (Mishina and Asakura, 1974; Koyama et al., 1982b) has been used to measure the blood flow velocity in the

Received for publication January 22, 1983

* With leave from Seishu Women College, Sapporo.

新田順子，小山富康，菊池佑二，進藤善雄 
peripheral and pulmonary microcirculations (KoYAMA et al., 1975, 1979; HоRIMOTo and KoYAMA, 1979, 1982; HoRimoto et al., 1979, 1981). However, since the method is based on the crossed-dual laser illumination of microvessels and the detection of weakly scattered light, its construction requires skillful adjustment of the optical system. An alternative method may be to use a grating laser and single beam illumination. The bidirectional optical system, i.e. the three-stage-prism grating microscope, has recently been tested by several authors (REUTER and TALUKDER, 1981; TAlukder and Reuter, 1981; SlaAf et al., 1981; KieSEWetter et al., 1982). The application of the prism grating seems to permit the on-line measurement of flow velocity of erythrocyte and even their direction. Simultaneous detection of bidirectionally focused images and subtraction of the projection images are advantageous for eliminating the so-called pedestal voltages and other noises (SLAAF et al., 1981). However, the precise three-stage-prism is not widely available and the signal processing system is complicated. In contrast, the grating microscope using a glass plate grating seems to be simpler in its optical construction and easier to use (USHIZAKA and ASAKURA, 1979; KOYAMA et al., 1982a). The image of a flowing erythrocyte is detected in this microscope by a photomultiplier through parallel windows in the grating. The time duration required for the erythrocyte to traverse the constant distance between adjacent windows (grating constant) is measured by an appropriate signal processing system. The flow velocity can be readily obtained, since it is proportional to the reciprocal of the time interval. The pedestal voltage is eliminated by means of electronic filters in the present system whereas it is done optically in the three-stage-prism method. However, it has been theoretically shown that particles whose velocity is to be measured must be smaller than the grating constant (USHIZAKA and AsaKura, 1979). If this measuring condition is essential, the optical parts of the grating microscope must be changed in relation to the size of particles to be measured. This requirement would reduce the usefulness of the grating microscope.

The first purpose of the present study is, therefore, the examination of this question. The performance of the grating microscope which was constructed in our laboratory using a simple glass grating (Ronchi grating) was tested with respect to the size of erythrocyte. The present study showed that the pedestal voltage induced by A. C. power could be easily eliminated by a high-pass filter and that reasonable information on flow velocity could be obtained in frog microvessels where large sized erythrocytes were flowing under oblique illumination. The second purpose was to study the effect of temperature on peripheral blood flow in poikilothermic vertebrates. The blood flow rate in the fish tail web, measured photoplethysmographically, showed no consistent increase in spite of warming (IRIKI and NAGAI, 1978). We were interested in knowing whether the inconsistent response of peripheral blood flow to whole body warming occurs also in other poikilothermic animals. The present experiments were designed to examine the effects of temperature changes on the flow velocity of erythrocytes in peripheral 
microcirculation by means of the new technique.

\section{PRINCIPLE AND METHODS}

The basic principle of the grating microscope is shown in Fig. 1. The direction of particle movement and the spatial grating surface are perpendicular to the optical axis of a microscope. The line of many windows in the grating is situated parallel to the direction of particle movement. A mask having a small aperture ( $1 \mathrm{~mm}$ in diameter) is placed just behind the grating in order to limit the probing area. The light transmitted through the mask is detected by a photodetector. The probing area of the fluid is illuminated at a small angle to the optical axis of the image detecting system to prevent the incident beam from entering the detector. The images of the moving erythrocyte are detected by the microscope. Their projection images on the grating reach the sensitive surface of a photomultiplier only when it passes through one of the windows of the grating. As a small particle moves within the fluid at a velocity $(V)$, it's image on the grating moves at a velocity $M V$, where $M$ is the magnification of the microscope. Then the image of the particle on the grating plane is hidden until it comes to the next window after a time period of $d / M V \sec$, where $d$, represents the grating constant $i . e$. the spacing between two adjacent windows. Since this sequence of events occurs repeatedly, the photomultiplier detects the image of the moving particle periodically at the time period given above. Thus, the translational motion of the particle is converted into an oscillating electrical wave signal with the wave period $T=d / M V$. From the measurement of $T$, the velocity of the particle is obtained by $V=d / M T$.

A laser grating microscope was constructed according to the description of

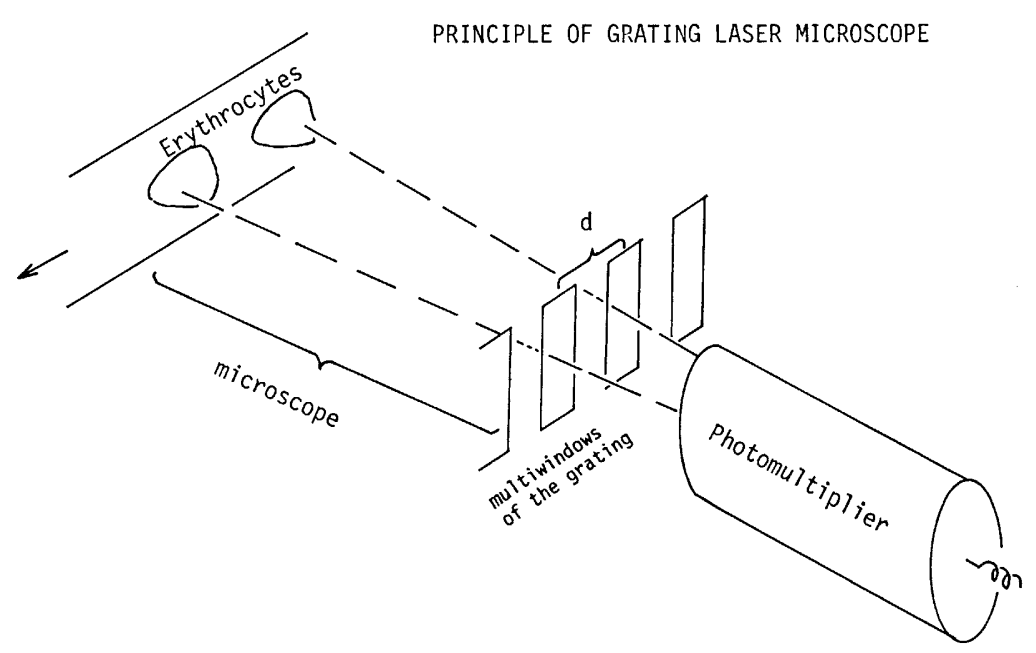

Fig. 1. Principle of the grating laser microscope.

Vol. 33, No. 3, 1983 


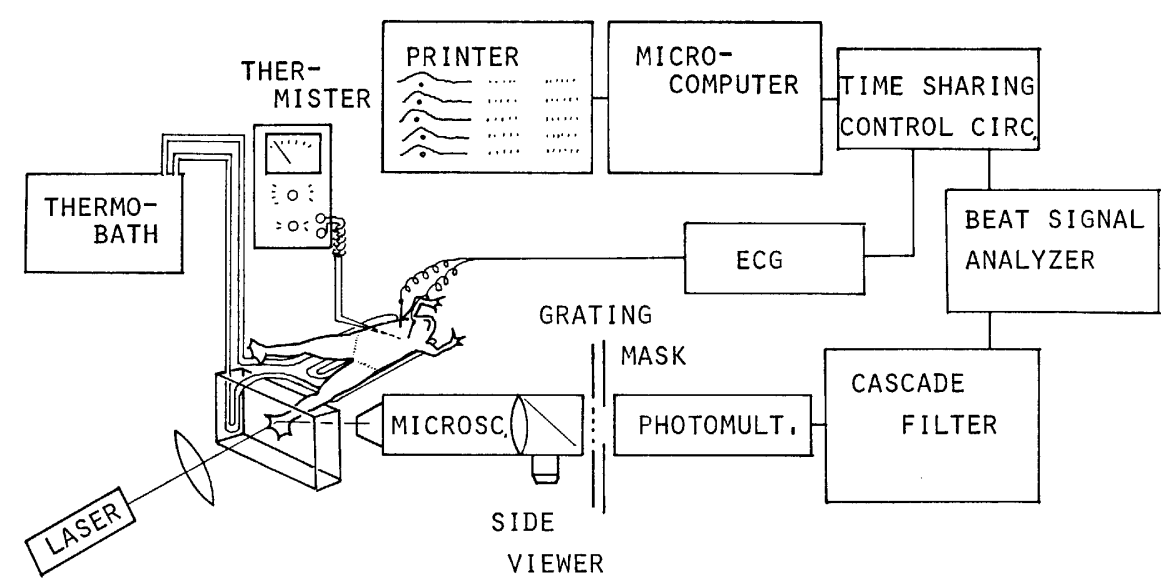

Fig. 2. A schematic representation of the grating laser microscope and experimental arrangement.

UshizaKa and AsaKura (1979). The arrangement of the grating microscope is shown schematically in Fig. 2. A He-Ne laser beam is focused on a small blood vessel by means of an objective lens (Olympus, $\times 10$ ) so that the illuminated area was $20 \mu \mathrm{m}$ in diameter. Erythrocytes are transilluminated obliquely by the thin laser beam with an incident angle of $45^{\circ}$. Images of erythrocytes are detected by a microscope having an objective lens $(\times 10)$ and an ocular lens (Olympus, eyepiece, $\times 10$ ). Images of erythrocytes are divided into two directions by means of a beam splitter; $86 \%$ for the photodetection to the photomultiplier and $14 \%$ for observation through the side viewer. A microflex system (Olympus, PM-6) formed a suitable beam splitter. Observation through the side viewer allows the projection of erythrocytes onto the image plane of the photomultiplier. A glass plate grating which has black straight lines at $100 \mu \mathrm{m}$ intervals (Ronchi grating) is used for the space grating. A magnified probing area contained ten black straight lines.

The output signals from the photomultiplier contain indices of the flow velocity. However, the signals oscillate because of slow fluctuations in photomultiplier noise and output voltages induced by ambient light and A. C. power supply i. e., the pedestal voltage. The output signals, therefore, are filtered by means of high and low pass filters (Cascade filter, FV-624, NF Co., Japan). The filtered signals result in a burst-like wave consisting of a small number of oscillations. They are clipped and transformed into square waves. Their wave periods are measured by means of calibration pulses at frequencies of $0.1,1$, and/or $10 \mathrm{MHz}$ generated by a beat signal analyzer (27-3000, Nihonkagaku Kogyo Co., Japan). Each measured wave period is compared with the next wave period. When they agreed within $12 \%$, the first of the two values is assumed to be valid. This comparison for validation of measured values is made sequentially. The valid wave 
period is stored in the memory of a microcomputer. The stored wave periods are arranged, so as to form a flow velocity appearance frequency histogram. To measure the pulsating flow velocity, measured wave periods were time-shared and stored in 16 channels arranged in chronological order after the occurrence of the R-wave of ECG. A total of $2 \times 10^{4}$ wave periods were pooled in 16 channels of the memory over a number of heart beats. Each channel covered the sequential time periods of $100 \mathrm{msec}$. The data accumulated in 16 channels formed 16 histograms of the wave period appearance frequency.

The performance of the present grating laser microscope was tested in vitro using a suspension of latex particle $(D=10 \mu \mathrm{m})$ flowing through a vertical glass tube $(D=8 \mathrm{~mm})$. The suspension contained latex particles at a rate of $0.1 \mathrm{ml} / \mathrm{liter}$ of water. The laser light for the transillumination was focused in the central portion of the tube. The image collecting microscope, which was focused in the central portion of the tube, was positioned in a plane containing the laser source and vertical to the streamline of the suspension, but with an angle of $45^{\circ}$ against the transilluminating laser beam. The outflow rate of the suspension was measured by means of a scaled cylinder. The flow velocity at the central portion of the tube was calculated assuming a parabolic profile for the flow velocity.

The images of an erythrocyte under oblique illumination were tested by microscopic observation of erythrocytes smeared on a glass plate. Fresh blood was centrifuged at $15,000 \mathrm{~g}$ for $5 \mathrm{~min}$. A small portion of erythrocyte column $(100 \mu \mathrm{l})$ was suspended in $10 \%$ glutharaldehyde phosphate Ringer's solution $(1 \mathrm{ml})$. The erythrocyte suspension was smeared on a cover glass and dried in room air. The cover glass with smeared erythrocytes was brought into a straight transillumination and into an illumination having an incident angle of $45^{\circ}$. The images of erythrocytes were magnified with an object lens $(\times 40)$ and eyepiece $(\times 10)$. The magnified images were photographed under the above two conditions of illumination.

Densely smeared erythrocytes were examined in another in vitro test. Glutharaldehyde suspension of erythrocytes was again centrifuged. Its erythrocyte portion was smeared on a glass plate. The magnified image of the densely smeared erythrocyte preparation was photographed under the microscope. The thickness of the smeared cells was measured by focusing the microscope on the glass plate surface and on the smeared cell surface. Then, the glass plate was attached to a motor which rotated at the rate of $1 / 4$ rotation per min, to check the flow velocity signals. Flow velocity signals which were induced by particles flowing through the probing area were recorded in a store recorder (DS-322, NF Co., Tokyo) at high speed and reproduced on a pen recorder at low speed.

An African clawed toad, Xenopus laevis (6 frogs weighing 20-60 g) was anaesthetized by immersion in a solution of MS222 (2.5 g/liter) and placed in a supine position on a tray mounted on a $x, y, z$-manipulator. To keep the frog wet during observation, one foot was dipped in a water chamber made of quartz and the other 
part of the body was covered with wet gauze. Two needle electrodes were inserted into the pectoral muscle on both sides of the heart to obtain ECG signals which were used to trigger for a time sharing control circuit. The center of a venule (diameter 20 to $60 \mu \mathrm{m}$ ) illuminated by the laser beam was adjusted to the center line of the side viewer to position the probing area on the aperture of the mask by controlling precisely the manipulator. In the present study, only the velocity along the center line of venules was measured. The temperature of the frog was raised by thermoregulated water circulating through a pipe embedded in the tray and placed in the water chamber for the foot. Temperature was measured by means of a thermocouple placed under the back of the frog (Fig. 2).

\section{RESULTS}

Examples of erythrocyte images photographed under straight transillumination and under transillumination at an incident angle of $45^{\circ}$ are shown in Fig. 3A and $\mathrm{B}$, respectively. The flat oval shape of the erythrocytes can be seen under straight transillumination, while only a thin fractional margin is visible using the $45^{\circ}$ illumination. The small bright area is a small fraction of the whole surface of the erythrocyte. The erythrocyte appears crescent-shaped when illuminated obliquely. A photographic recording of the densely-smeared erythrocyte preparation is shown in Fig. 4. Its thickness was $8 \mu \mathrm{m}$ and many small pores and their bright edges were present among the densely-smeared erythrocytes. Burst-like oscillations of the photomultiplier output were produced by moving erythrocytes in both the diffusely and densely-smeared preparations. Examples of such signals from the densely-smeared erythrocyte preparation are shown in Fig. 5.

Smooth and clear oscillations in output signals were obtained from moving particles in a glass tube as shown in Fig. 6, which were recorded at two velocities, whose wave period becomes shorter with each increment in velocity. The relation between their wave period and the center line flow velocity calculated from the flow rate through the glass tube is given in Fig. 7A. It can be seen that the wave period was inversely proportional to the center line flow velocity. The center line flow velocity which was determined by putting the measured wave period and the value of 55 for $M$ into the above equation is plotted on the ordinate in Fig. 7B. This value was used for $M$ in the calculation of erythrocyte flow velocity in the present in vivo test.

Burst-like signals could be obtained in the blood vessels of diameter 20 to $60 \mu \mathrm{m}$ in vivo. The velocity signal in capillary blood vessels in vivo was low in signal to noise ratio. But still clear burst-like signals could be obtained from web capillaries as shown in Fig. 8.

An example of the final output from the data processing system is shown in Fig. 9, which was obtained in a venule having a diameter of $40 \mu \mathrm{m}$ in a frog web. Appearance histograms of wave periods were stacked in order according to the lapse 


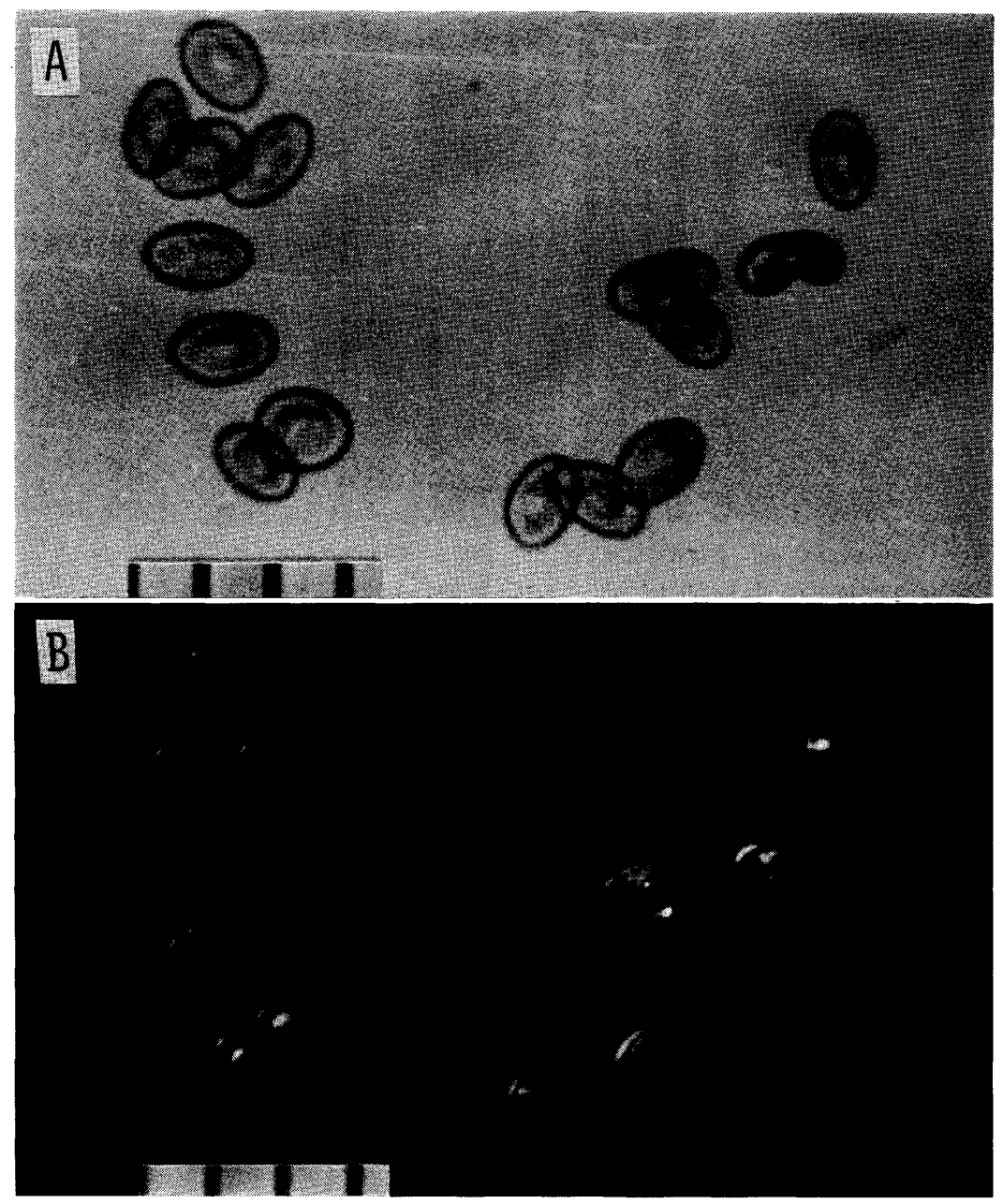

Fig. 3. Photographic images of frog erythrocytes diffusely smeared on a glass plate. A, straight transillumination: B, oblique illumination. The scale shows $10 \mu \mathrm{m}$.

of time for each $100 \mathrm{msec}$ after the occurrence of the R-wave of the ECG. The mean value for each histogram, which is the most probable value for each time section, was calculated and indicated on each histogram. Their numerical values are typed in the left column and the average velocities with standard deviatoin in the right column. The curve following the marked mean values from the bottom to the top shows the flow velocity contour during the cardiac cycle. It is noteworthy that the position of mean values on the histograms changed with time. In the present study the flow velocity contour has a minimum at 2 to $4 \times 10^{2} \mathrm{msec}$ and a maximum at 8 to $10 \times 10^{2} \mathrm{msec}$ after the $\mathrm{R}$-wave.

Heart rate and blood flow velocity are plotted against temperature in Figs. 10 


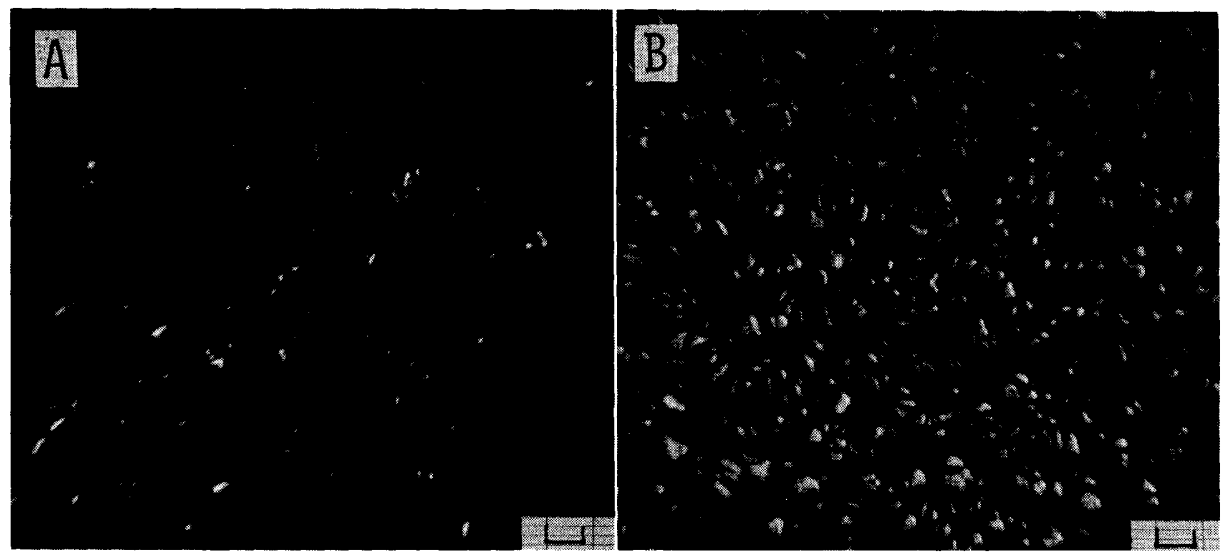

Fig. 4. Photographic images of frog erythrocytes densely smeared on a glass plate. Small bright points can be seen between erythrocytes in A. A: oblique transillumination, B: straight transillumination. The scale shows $10 \mu \mathrm{m}$.

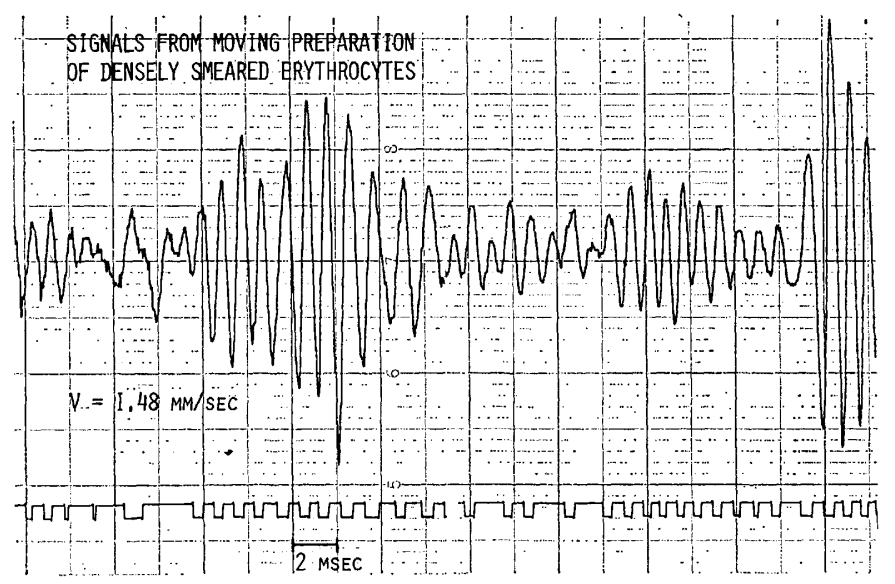

Fig. 5. Examples of burst-like oscillations in output signals of the photomultiplier. The preparation of the densely smeared erythrocytes was moving at the velocity of 1.48 $\mathrm{mm} / \mathrm{sec}$.

and 11, respectively. Heart rate increased clearly with the rise in temperature, while flow velocity showed inconsistent responses.

\section{DISCUSSION}

If the particle is small enough and if only one particle exists in the probing area, its image can be seen only when it traverses windows. The intensity of the scattered light, therefore, is repeatedly low and high as the particle traverses the pro- 
SIGNALS FROM POLYSTYRENE SUSPENSION FLOWING THROUGH GLASS TUBE
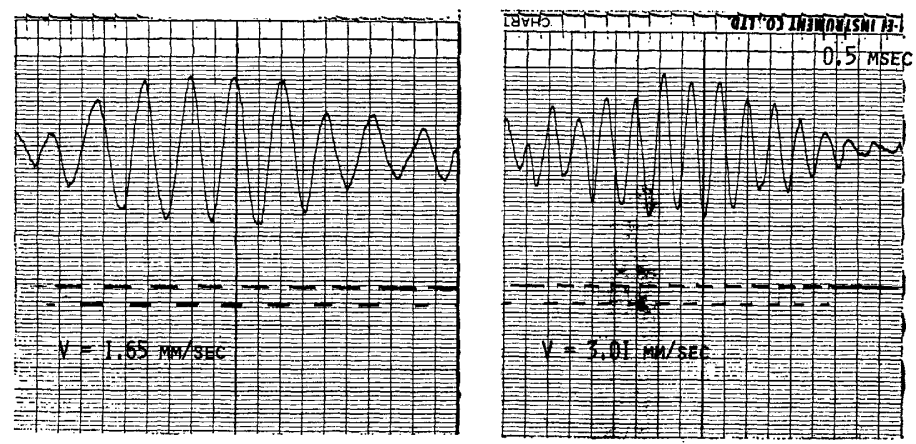

Fig. 6. Examples of burst-like oscillations obtained from polystyrene particles flowing through a glass tube at two flow velocities. Curves from top to bottom represent timer, burst-like oscillations and square waves for wave period measurements.

A

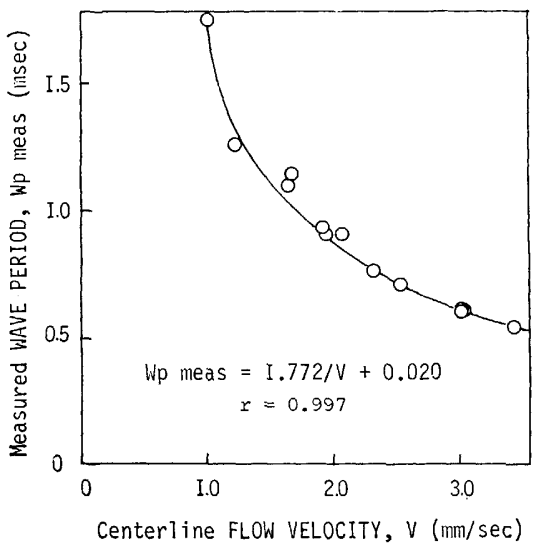

B

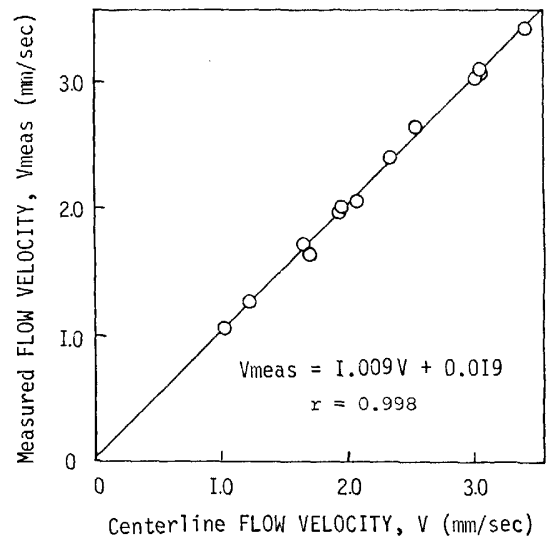

Fig. 7. A: relation between the wave period of the oscillations in the output signals from the grating laser microscope and center line flow velocity estimated from the flow rate measured by a scaled cylinder. B: the flow velocity calculated with the assumption of $M=55$ and the center line flow velocity estimated from the flow rate.

bing area. This clear oscillation in light intensity is advantageous for velocity measurement. However, a theoretical analysis on the performance of the grating microscope (UsHIZAKA and ASAKURA, 1979) concluded that the detection of flow velocity becomes difficult when the size of particle images exceeds the grating constant. Human and frog erythrocytes exceeded this size limit in the present study. In spite of their large size, however, well defined burst-like signals were observed both in vitro and in vivo (see Figs. 5 and 6). To understand these results, photographic recordings of erythrocyte images during the in vitro test were inspected. 


\section{SIGNALS FROM AN ERYTHROCYTE \\ FLOWING THROUGH A CAPILLARY IN VIVO}

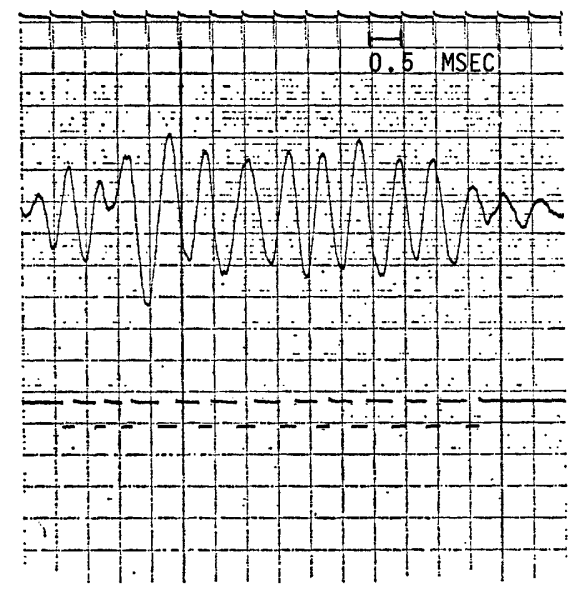

Fig. 8. An example of burst-like oscillation signals from an erythrocyte flowing in a web capillary.
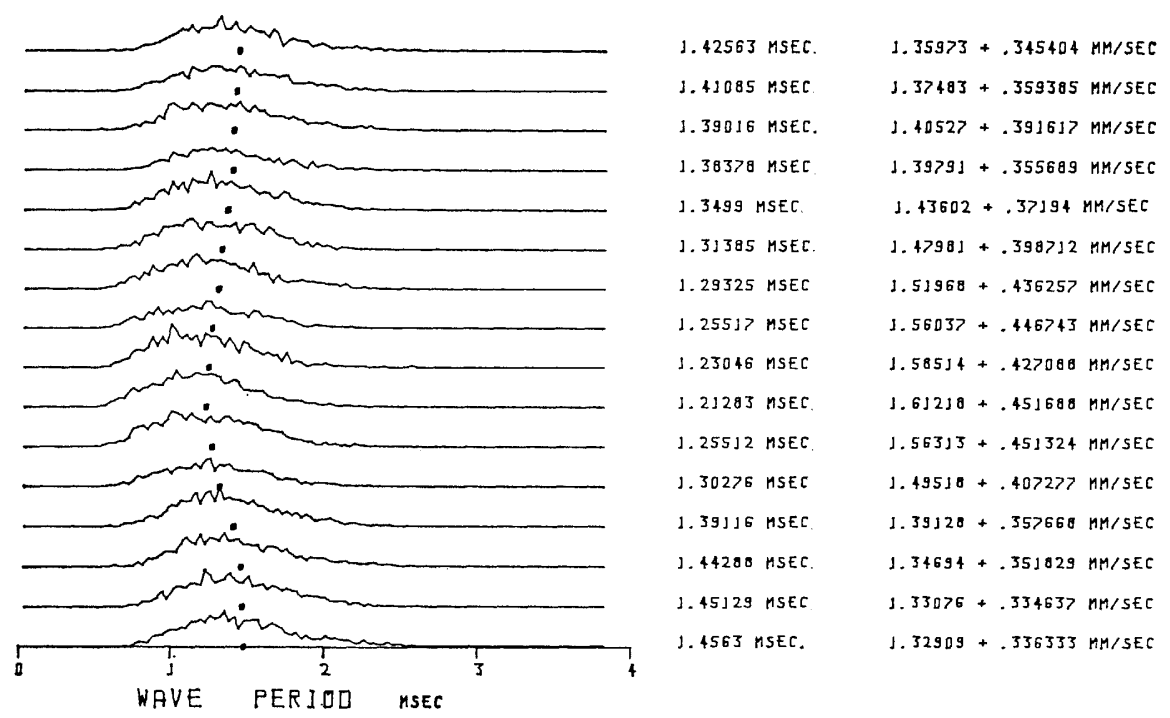

Fig. 9. An example of the final output from the data processing system.

This suggested (Fig. 3) the possibility that only a small fraction of the total cell area is intensely illuminated, as it flows in an obliquely illuminated probing area in vivo. The magnified image of these small fractional areas does not exceed the grating constant. Thus, the flow velocity of human and frog erythrocytes whose 


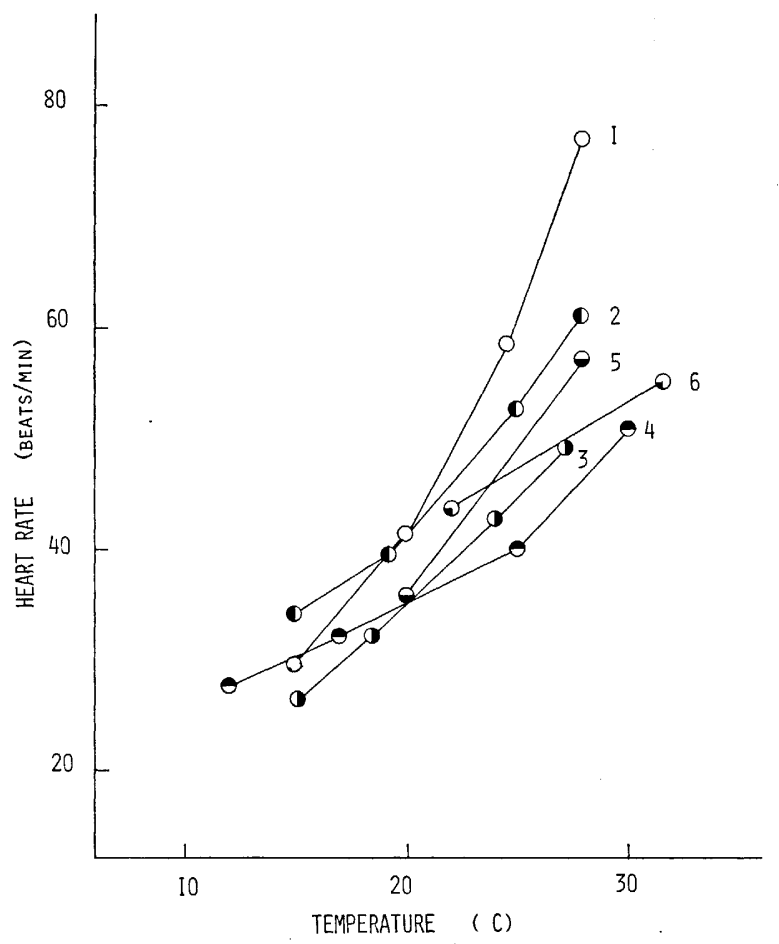

Fig. 10. Relation between heart rate and body temperature for 6 individual frogs.

diameter is large relative to the grating constant can be readily measured by means of the present system. In fact, burst-like oscillations of output signals were recorded from moving erythrocytes both in vitro and in vivo. Burst-like oscillating signals were obtained also in the densely smeared erythrocyte preparation (see Fig. 5). This unexpected but advantageous result can be explained by the photograph shown in Fig. 4. Small spaces remained between erythrocytes and formed illuminated spots. As the glass plate rotates, the bright regions move and cause periodic changes in the total intensity of the output signals. A simple calculation assuming a haematocrit value of $40 \%$ and the erythrocyte size of $13.1 \times 8.5 \mu \mathrm{m}$ suggests that spacings of adequate size ( 2 to $3 \mu \mathrm{m}$ ) can be formed at a certain probability in vessels of 20 to $60 \mu \mathrm{m}$ in diameter.

The wave period of the oscillation was inversely proportional to the center line flow velocity estimated from the flow rate measured by means of a scaled cylinder. The calibration curve of flow velocity showed a proportional relation. Various values were assumed for $M$ in the calculation of flow velocity. An $M$-value of 55 yielded the best fit regression line. Although the regression line deviated slightly from the diagonal, it is apparent that the flow velocity can be measured within a $3 \%$ error. The range of the flow velocity shown in Fig. 7 was selected so as to 


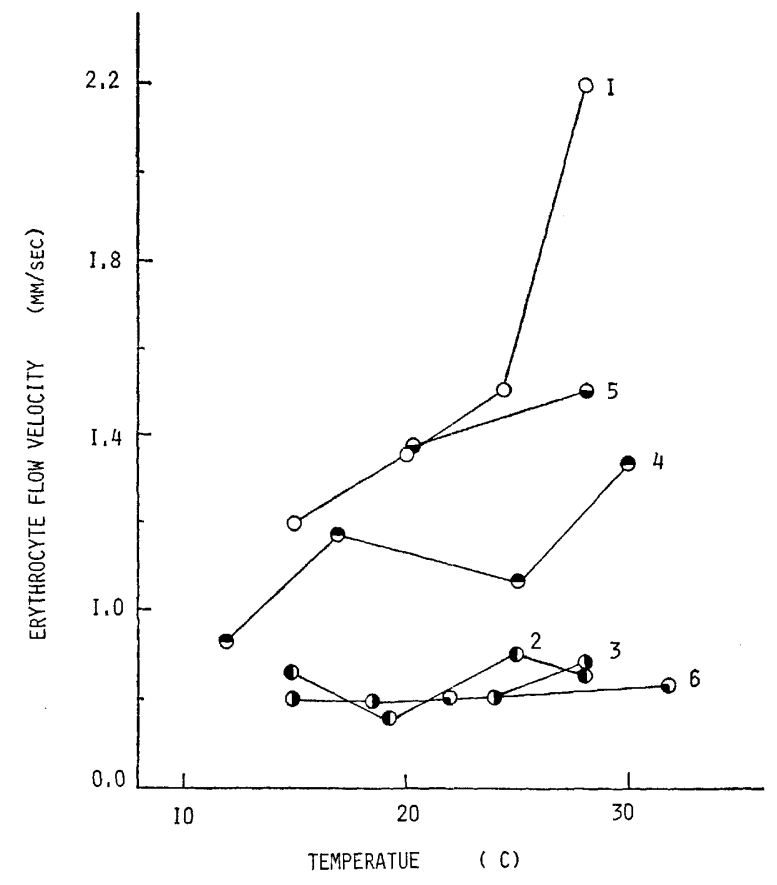

Fig. 11. Relation between erythrocyte flow velocity and temperature for each of the frogs studied.

cover the flow velocity in microvessels. It was seen that a flow velocity higher than $10 \mathrm{~cm} / \mathrm{sec}$ could be measured by the present method.

Theoretically an incoherent light beam may be used in a grating microscope. However, as a matter of practice, a laser beam is advantageous, because its diameter can be easily reduced and the amount of the light reflected on the tissue surface surrounding the probing area can be decreased. The decrease in the reflected light greatly improves the signal-noise ratio for the velocity signals.

The wave period of the burst-like oscillating signals in capillaries were often nonuniform and interrupted by a short wave period (Fig. 8). It can be observed under the microscope that erythrocytes deform when they flow in capillaries. Probably the bright points on the edge of an erythrocyte change their position during its passage through the probing area. Such an irregular wave period was discarded from the velocity data by comparing it with the preceding wave period. The rate of data adoptation in the in vivo measurements was about $40 \%$.

The blood flow velocity in venules pulsated with the heart beat. It began to increase at 500 to $600 \mathrm{msec}$ after the R-wave of ECG and attained a maximum value at $900 \mathrm{msec}$. This lag time is much longer than for arterioles (HoRImoto et al., 1979). The pulsation amplitude was $25 \%$ of the mean flow velocity at most. These features are probably typical of venules. The present results suggest that 
the grating laser microscope is a suitable instrument for detecting pulsations in blood flow velocity.

Metabolic rate and other physiological parameters are doubled by each $10^{\circ} \mathrm{C}$ rise in temperature within the viable temperature range of poikilothermic animals (SCHMidT-NiELSEN, 1975). Consistent with this general concept the heart rate in all frogs studied increased at a high rate. However, the flow velocity in web venules increased with temperature only in frogs Nos. 1, 4, and 5 and at a rate which was much less than that of the heart rate. Moreover, in frogs Nos. 2, 3, and 6 flow velocity remained almost unaffected. When heart rate increases with a rise in temperature, cardiac output will probably be increased. An increased cardiac output would result in a rise in flow rate in the peripheral microvessels. This expectation, however, was not confirmed by the present measurements shown in Fig. 11. The present result obtained for frog web was similar to the observation previously reported on blood volume in the fish tail web(IrIKI and NAGAI, 1978). The blood flow rate to the other visceral organs will be increased in response to the metabolic activities intensified by the rise in temperature. However, any redistribution of cardiac output to the important organs would reduce any increment in the flow rate through some peripheral microvessels such as those in the web of feet. The extent to which such redistribution occurs will depend on many factors and perhaps accounts for the variability in results obtained in the present experiments. The peripheral blood flow velocity is affected by various intrinsic fac ors in a complicated way in relation with temperature.

It is concluded that the present grating laser microscope can provide us ful information on the peripheral microcirculation. The optical arrangement is imple and mechanically stable. Its probing area is easily selected through the side viewer.

The authors wish to express their thanks to Prof. T. Asakura and Mr. T. Ushizaka for the valuable suggestions. They are much indebted to Prof. G. M. Hughes, University of Bristol, England for his comments and encouragements. They wish also to thank Mr. T. Arai for his skilfull aid in the construction of the grating laser microscope. The frogs were applied by courtesy of the Zoological Institute of Hokkaido University.

\section{REFERENCES}

Horimoto, M. and Koyama, T. (1979) Pulsatile blood flow in arteriole of frog web. Biorheology, 16: $163-170$.

Horimoto, M. and Koyama, T. (1982) Acute microcirculatory response to nicotine in frog web. Jpn. J. Physiol., 32: 771-782.

Horimoto, M., Koyama, T., Kikuchi, Y., KaKiuchi, Y., and Murao, M. (1981) Effect of transpulmonary pressure on blood flow velocity in pulmonary microvessels. Respir. Physiol., 42: 31-41.

Horimoto, M., Koyama, T., Mishina, H., Asakura, T., and Murao, M. (1979) Blood flow velocity in pulmonary microvessels of bullfrog. Respir. Physiol., 37: 45-59.

IRIKI, M. and NAGAI, M. (1978) Temperature stimulation and peripheral blood flow in teleostes. Bull. Physiol. Soc. Jpn., 40: 363 (in Japanese). 
Kiesewetter, H., RadTke, H., Korber, N., and Schmid-Schönbein, H. (1982) Experimental calibration of a two-stage prism-grating system for measuring cell velocity. Microvasc. Res., 23: 56-66.

Koyama, T., Asakura, T., Nitta, J., Kikuchi, Y., Arai, T., Ushizaka, T., Tsushima, N., and KAKIUCHI, Y. (1982a) Flow Velocity Measurements in Basic Aspects of Microcirculation, ed. by TsuchiYa, M. and Asano, M. Excerpta Medica, Amsterdam-Oxford-Princeton, pp. 31-38.

Koyama, T., Horimoto, M., Mishina, H., and Asakura, T. (1982b) Measurements of blood flow velocity by means of a laser Doppler microscope. Optik, 61: 411-426.

Koyama, T., Horimoto, M., Mishina, M., Asakura, T., Horimoto, M., and Murao, M. (1979) Laser Doppler microscope in an oblique-backward mode and pulsatile blood flow velocity in pulmonary arteriole. Experientia, 36: 65-66.

Koyama, T., Mishina, H., and Asakura, T. (1975) A study of micro-circulation in web of frog by using laser Doppler microscope. Experientia, 31: 1420-1421.

Mishina, H. and Asakura, T. (1974) A laser Doppler microscope. Opt. Commun., 11: 99-102.

Reuter, B. and TAlukder, N. (1981) New differential laser microanemometer. Proc. Soc. Photo-opt. Instrum. Eng., 236: 226-230.

SCHMidT-Nielsen, K. (1975) Animal Physiology (Adaptation and Environment), Cambridge Univ. Press, London, pp. 260-269.

SlaAf, D. W., Rood, J. P. S. M., Tangelder, G. J., Jeurens, T. J. M., Alewijnse, R., Reneman, R. S., and ARTS, T. (1981) Microvasc. Res., 22: 110-122.

TAlUKDeR, N. and ReUter, B. (1981) Messung von Wandeinflüssen auf die Strömung makromolekulärer Flüssigkeiten mit Hilfe des Laser-Differential-Mikroanemoimeters. Biomed. Tech., 26: Ergänzungsband 120-121.

UshizaKA, T. and AsakuRA, T. (1979) A measuring method of flow velocity in a microscopic area using a grating spatial filter. Bull. Res. Inst. Appl. Electr., Hokkaido Univ., 32: 15-32, 1980. 